\title{
THE TOTAL CURVATURE OF KNOTTED SPHERES
}

\author{
BY DAN SUNDAY
}

Communicated December 2, 1974

Chern and Lashof [1] proved several inequalities concerning the total curvature of an immersed manifold. Their second result is a weak generalization of the Fary-Milnor theorem [2] , [5] for closed space curves. In this paper, a stronger result (Corollary 1), the complete homotopy extension, is stated and proved. I would like to thank Bill Pohl for conversations surrounding the formulation and proof of this result.

I. Background. Let $x: M^{n} \rightarrow E^{n+N}$ be a $C^{\infty}$-immersion into Euclidean space of dimension $n+N(N>0)$; and $B_{\nu}$ be the bundle of unit normal vectors of $x\left(M^{n}\right)$. A point of $B_{\nu}$ is a pair $(p, \nu(p))$, where $\nu(p)$ is a unit normal vector to $x\left(M^{n}\right)$ at $x(p)$. The map $\bar{\nu}: B_{\nu} \rightarrow S_{0}^{n+N-1}$, into the unit sphere of $E^{n+N}$, is defined by $\bar{\nu}(p, \nu(p))=\nu(p)$.

The Lipschitz-Killing curvature [1] $G(p, \nu)$ at $\nu(p)$, is then given by the $\bar{\nu}$. ratio of corresponding volume elements in $S_{0}^{n+N-1}$ and $B_{\nu}$. The total curvature of $M^{n}$ at $p$ is $K^{*}(p)=\int|G(p, v)| d \sigma$, the integral being taken over the sphere of unit normal vectors at $x(p)$. The total curvature of $M^{n}$ is given by $K^{*}=K^{*}(M)$ $=\int_{p \in M} K^{*}(p) d V$.

The first two Chern-Lashof theorems can be stated as: Given $M^{n}$ compact without boundary, and $c(m)$ the area of the unit hypersphere $S_{0}^{m} \subset E^{m+1}$, then:

Corollary 1. $K^{*}(M) \geqslant 2 c(n+N-1)$.

Corollary 2. If $K^{*}(M)<3 c(n+N-1)$, then $M$ is homeomorphic to $S^{n}$.

The essential argument of their proof can be summarized as a lemma.

LEMma 1. If, for almost all $v_{0} \in S_{0}^{n+N-1}$, the height function $\left\langle v_{0},-\right\rangle$ : $x(M) \rightarrow R$ has at least $k$ distinct critical points, then $K^{*}(M) \geqslant k c(n+N-1)$.

Their method is an adaptation of the technique used by Fenchel [3]. This fact suggested that Corollary 2 is a weak generalization of Fary-Milnor.

II. The main result. In this section, a curvature inequality is given which distinguishes between different knottings of $S^{n}$. The method, based on Chern-Lashof, takes off from a remark of Fox [4] in which P. L. approximations yield the corresponding $S^{1}$ result.

AMS (MOS) subject classifications (1970). Primary 53C65; Secondary 57C45, 57 D40.

Key words and phrases. Fary-Milnor theorem, normal bundle, Gauss map, knot group, Morse equality. 
For simplicity of presentation, attention is restricted to knotted spheres; that is, $M^{n}=S^{n}$ and codimension $N=2$. Recall, for a mapping $x: S^{n} \rightarrow$ $E^{n+2}$, the group of the map is $\pi(x)=\pi_{1}\left[E^{n+2}-x\left(S^{n}\right)\right]$.

DEFINITION 1. $g(x)=$ the minimal number of generators needed to present $\pi(x)$.

THEOREM I. $K^{*}\left(S^{n}\right) \geqslant 2 g(x) c(n+1)$.

Corollary 1. If $K^{*}\left(S^{n}\right)<4 c(n+1)$, then $\pi(x)=Z$.

The corollary follows trivially since any $\pi(x)$ has $Z$ as a subgroup. Theorem $I$ is a consequence of Lemma 1 combined with the obvious.

Proposition 1. For almost all $v_{0} \in S_{0}^{n+1}$, the height function $\left\langle v_{0},-\right\rangle$ : $x\left(S^{n}\right) \rightarrow R$ has at least $2 g(x)$ distinct critical points.

Proof. Since we only need to account for an open dense subset of the $v_{0}$ 's, fix a height $\left\langle v_{0},-\right\rangle$ which is Morse. Choose a basepoint, *, which is "higher" than $x\left(S^{n}\right)$. The proposition is shown by constructing a canonical set of generators for $\pi\left(x,{ }^{*}\right)$, and deforming an arbitrary loop, $\gamma \in \pi\left(x,{ }^{*}\right)$, into a sum of these. The deformation is first described. The required generating set will be obvious at the outcome.

Since ${ }^{*}$ is higher than $x\left(S^{n}\right)$, assume that the loop $\gamma$ is strictly lower than ${ }^{*}$. Now, define a lifting-homotopy as a homotopy $H(x, t)$ which is always moving to higher levels, that is one where $\left\langle v_{0}, H(x, t)\right\rangle$ is nondecreasing in $t$ for all fixed $x$ in the loop parametrization. The problem involved is to determine the obstructions in $x\left(S^{n}\right)$ preventing $\gamma$ from being pulled up all the way. Clearly, any such phenomenon will be local. The crucial observation is that $\gamma$ can only be "caught" on maximums of $\left\langle v_{0},-\right\rangle: x\left(S^{n}\right) \rightarrow R$.

Take a collection of open collared balls, $U_{i} \subset W_{i}$, in $E^{n+2}$ such that: (1) $\left\{U_{i}\right\}$ is a finite covering of a simply-connected volume enclosing $x\left(S^{n}\right)$; (2) each critical point $p$ is contained in only one ball $W_{i}$; and (3) there are Morse-coordinates for $\left(W_{i} \cap x\left(S^{n}\right)\right)$ whose axes are strictly monotonic w.r.t $\left\langle v_{0},-\right\rangle$. Clearly, any part of $\gamma$ lying in a $U_{i}$ not containing a critical point can be lifted out of the ball. This means that attention can be focused on the $U_{1}, \ldots, U_{k}$ containing $p_{1}, \ldots, p_{k}$.

Now, suppose that $p_{j}$ is not a maximum. Then the height function is increasing on at least one Morse-axis, and the piece of $x\left(S^{n}\right)$ locally obstructing $\gamma$ has at least codim 3. There are index $\left(p_{j}\right)>0$ degrees of freedom with which to translate a segment of $\gamma$ and lift it into the collar $\left(W_{j}-U_{j}\right)$ such that it lies above $U_{j} \cap x\left(S^{n}\right)$. After a finite number of such movements, $\gamma$ will only be obstructed by balls containing maximums.

Next, assign a unique 'canonical' element of $\pi(x)$ to each maximum. For $p_{j}$ a maximum, fix a loop $\gamma_{j}$ which passes under $p_{j}$ only once. This can be ar- 
ranged (inside $W_{j}$ ) by adding a lower hemisphere to $U_{j} \cap x\left(S^{n}\right)$, and taking $\gamma_{j}$ as a generator which leaves $W_{j}$ through the north pole and is increasing till $*$. Any segments of $\gamma$ stuck in $U_{j}$ can be lined up (inside $W_{j}$ ) with $\gamma_{j}$. The rest of the loop goes up and away. Hence, the collection $\left\{\gamma_{j}\right\}$ is a set of generators for $\pi(x)$.

Summarizing, any $\left\langle v_{0},-\right\rangle$ has at least $g(x)$ maximums. Next, if $C_{i}=$ the number of critical points of index $i$, then the Morse equality gives: (1) for $n$ odd, $\Sigma_{i=1}^{n}(-1)^{i+1} C_{i}=C_{0} \geqslant g(x)$, and there are at least $g(x)$ critical points other than maximums; (2) for $n$ even, there is at least one minimum, hence: $\sum_{i=1}^{n=1}(-1)^{i+1} C_{i}=C_{0}+C_{n}-2$, and there are at least $(g(x)-1)$ critical points other than maximums and minimums. In either case, the proof is complete.

\section{BIBLIOGRAPHY}

1. S. Chern and R. K. Lashof, On the total curvature of immersed manifolds, Amer. J. Math. 79 (1957), 306-318. MR 18, 927.

2. I. Fáry, Sur la courbure totale d'une courbe gauche faisant un noeud, Bull. Soc. Math. France 77 (1949), 128-138. MR 11, 393

3. W. Fenchel, On the differential geometry of closed space curves, Bull. Amer. Math. Soc. 57 (1951), 44-54. MR 12, 634.

4. R. H. Fox, On the total curvature of some tame knots, Ann. of Math. (2) 52 (1950), 258-260. MR 12, 273.

5. J. W. Milnor, On the total curvature of knots, Ann. of Math. (2) 52 (1950), 248257. MR 12, 273.

DEPARTMENT OF PHYSIOLOGY AND ANATOMY, UNIVERSITY OF CALIFORNIA, BERKELY, CALIFORNIA 94720 